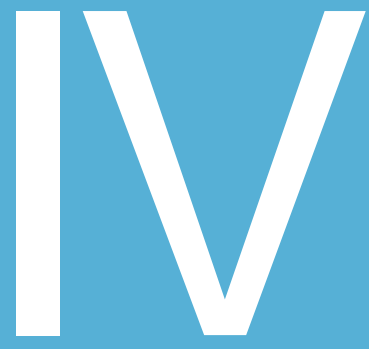

\title{
PARENTALIDAD Y AUTOESTIMA DE LOS HIJOS: UNA REVISIÓN SOBRE LA IMPORTANCIA DEL FORTALECIMIENTO FAMILIAR PARA EL DESARROLLO INFANTIL POSITIVO
}

\section{Parentality and children's self-esteem: A revision over the importance of family strengthening for the positive development of children}

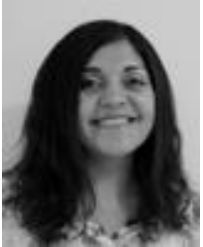

\section{Jael Alejandra Vargas Rubilar}

Licenciada en Psicología. Becaria Doctoral CIIPME- CONICET Centro de Investigación en Psicología y Ciencias Afines de la Universidad Adventista del Plata, funcionando como Grupo Vinculado (Resolución por parte del CONICET № 0018/10) al Centro Interdisciplinario de Investigaciones en Psicología Matemática y Experimental. Libertador San Martín, Entre Ríos, Argentina

\section{Laura Beatriz Oros}

Licenciada en Psicología, Universidad Adventista del Plata. Doctora en Psicología, Universidad Nacional de San Luis. (Doctorado acreditado por la Comisión Nacional de Evaluación y Acreditación Universitaria CONEAU). Centro de Investigación en Psicología y Ciencias Afines de la Universidad Adventista del Plata, funcionando como Grupo Vinculado (Resolución por parte del CONICET № 0018/10) al Centro Interdisciplinario de Investigaciones en Psicología Matemática y Experimental. Libertador San Martín, Entre Ríos, Argentina. 


\section{Resumen}

La autoestima positiva promueve la salud y protege de la disfuncionalidad, emergiendo como un importante factor de resiliencia. Su desarrollo, desde edades tempranas, favorece una adaptación funcional al medio y previene el disconfort emocional a largo plazo. Dado que la literatura afirma que la familia es una pieza clave para la génesis de la autoestima infantil, el presente trabajo reseñará algunas condiciones familiares que pueden afectar la valoración personal de los hijos, remarcando la necesidad de diseñar abordajes psicosociales para propiciar ambientes familiares salugénicos, optimizando las competencias parentales y fortaleciendo el vínculo afectivo parento-filial.

Palabras clave: autoestima infantil; familia; intervención psicosocial, padres.

\section{Abstract}

Positive self-esteem promotes health and protects against dysfunctionality, emerging as an important factor of resilience. Its development in early stages favors a functional adaptation to the environment and prevents emotional discomfort in the long term. Since literature states that a family is a key factor for the creation of children's self esteem, this research will review some family conditions that can affect the personal assessment of the children, highlighting the necessity of creating psychosocial approaches for the creation of healthy family environments, optimizing the parental competences and strengthening the affective bond between parents and children.

Key words: children's self-esteem; family; psychosocial intervention 


\section{Introducción}

Los avances realizados por la Psicología positiva en los últimos años, han permitido identificar las fortalezas personales que hacen posible resistir a la adversidad y desarrollarse a pesar de vivir en condiciones de vida adversas, un fenómeno también conocido como resiliencia. La autovaloración o autoestima positiva ha sido considerada por investigadores clásicos como una de las características centrales de las personas resilientes (Kobasa, 1985; Rutter, 1985, Walsh, 2004). Asimismo, importantes estudios la han mencionado entre los indicadores claves de salud mental, calidad de vida, bienestar, e incluso como ingrediente indispensable para la felicidad humana (Falcone Castillo et al., 2009; Fiorentino, 2008; Frías, Mestre y Del Barrio, 1990; Greco, Morelato e Ison, 2006; Oros y Richaud de Minzi, en prensa; Rutter, 1985; Seligman, 1998)

Dichos aportes han posibilitado analizar los recursos psicológicos no sólo desde un punto de vista teórico, sino además afianzar intervenciones que se focalicen en la promoción y fortalecimiento de factores protectores, como la autoestima, ante las problemáticas individuales y familiares.

Aunque en ocasiones se ha utilizado el término autoestima como intercambiable con el de autoconcepto, ambos constructos son claramente diferenciables, al menos desde el punto de vista teórico. El autoconcepto puede ser definido como la imagen o percepción descriptiva que uno tiene sí mismo; como un juicio reflexivo acerca de las propias capacidades, valores, preferencias o apariencias (González-Pienda, Pérez, Glez.-Pumariega y García García, 1997; Heathertorn y Wyland, 2003), y es considerado, por lo tanto, como un proceso predominantemente cognitivo (Garaigordobil y Durá, 2006). Por el contrario, la autoestima es la valoración, de fuerte connotación afectiva, que la persona atribuye a la descripción de sus características (Ramírez Peradotto, Duarte Vargas y Muñoz Valdivia, 2005; Gázquez, Pérez, Ruiz, Miras y Vicente, 2006). En este sentido, se puede decir que la autoestima comprende el componente valorativo y afectivo del autoconcepto (Cardenal y Fierro, 2004; Garaigordobil y Durá, 2006; Garaigordobil, Pérez y Mozaz, 2008); es una respuesta emocional que se experimenta al contemplar y evaluar diferentes aspectos de uno mismo (Heathertorn y Wyland, 2003). Existen otras perspectivas y enfoques de la autoestima (Mruk, 2006); ésta no es la única aproximación posible, pero es la que adoptaremos en el presente trabajo.

La autoestima puede considerarse un constructo tanto global como multidimensional. Desde el punto de vista global, se refiere a una cualidad generalizada que permea todos los aspectos vitales del individuo; 
mientras que desde una aproximación multidimensional, la autoestima se refiere a aspectos específicos del sí mismo, pudiendo desagregarse en autoestima de rendimiento, autoestima social y autoestima física (Heathertorn y Wyland, 2003), en sentimientos de capacidad personal y sentimientos de valía personal (Gázquez, Pérez, Ruiz, Miras y Vicente, 2006) o en otra variedad de dimensiones, dependiendo del enfoque teórico que se adopte y del contexto cultural en el cual se desarrollen los conceptos, medidas y modelos.

De acuerdo a Hewitt (2005), la autoestima es una emoción construida socialmente y, por lo tanto, muy influenciable por el contexto cultural. Desde la perspectiva de este autor, la autoestima, por ser una emoción, puede manejarse y autorregularse según las circunstancias sociales que tomen lugar, tal como se haría con otras emociones como la alegría, el enojo o el miedo.

Teniendo en cuenta estas aportaciones, no resulta extraño que algunos investigadores hayan vinculado la autoestima con las emociones positivas. El sentimiento de orgullo por las propias capacidades y logros y el sentimiento de valía personal y satisfacción por uno mismo son elementos íntimamente ligados a la autoestima positiva (Fredrickson, 2004; Oros y Richaud de Minzi, en prensa). El estudio de las emociones positivas, así como de otros factores favorecedores de una buena salud mental, viene cobrando gran atención en los últimos años gracias a los avances que ha realizado la Psicologia Positiva en materia de investigación empírica. A continuación se puntualizarán algunos beneficios que reportan la autoestima positiva y los sentimientos de orgullo y satisfacción personal.

\section{Ganancias de una autoestima positiva}

La autoestima ha sido relacionada con una gran cantidad de variables psicológicas y, aunque no todos los resultados son concluyentes en el mismo sentido, la gran mayoría de los estudios sugieren que la autoestima positiva se relaciona con una mejor salud mental y un mayor bienestar. Estudios realizados con niños y adolescentes han encontrado relaciones significativas entre la autoestima positiva y la cooperación, las habilidades sociales, la felicidad, la tolerancia al estrés, la flexibilidad, la adaptación a los cambios, la integración social, la capacidad de trabajar en equipo, la constancia (Garaigordobil y Durá, 2006), la planificación de respuestas (Gázquez, Pérez, Ruiz, Miras y Vicente, 2006), la percepción y el buen manejo del estrés (Verduzco Álvarez-Icaza, 2006) y la satisfacción con la vida (Martínez-Antón, Buelga y Cava, 2007), entre otros constructos. Específicamente con relación al sentimiento 
de orgullo personal, se ha sugerido que puede actuar como un poderoso motivador de los comportamientos prosociales y morales (Hart y Matsuba, 2007) y fomentar la perseverancia en tareas socialmente valoradas (Williams \& DeSteno, 2008), la atracción social y el ejercicio de roles dominantes en un grupo de trabajo (Williams \& DeSteno, 2009).

Por el contrario, la autoestima global débil, o alguna de sus dimensiones, se ha visto asociada a síntomas de depresión (Bragado, Hernández LLoreda, Sánchez-Bernardos y Urbano, 2008; Abela y Skitch, 2007; Orth, Robins y Roberts, 2008), tristeza (Ciarrochi, Heaven y Davies, 2007), impulsividad, celos, desajuste emocional (Garaigordobil y Durá, 2006), diversos síntomas psicopatológicos (Garaigordobil, Pérez y Mozas, 2008), problemas escolares (Navarro, Tomás y Oliver, 2006), conductas de riesgo (Musitu, Jiménez y Murgui, 2007) y, al igual que la autoestima exagerada, a conductas agresivas y violentas (Cava, Musitu y Murgui, 2006; Diamantopoulou, Rydell y Henricsson, 2008).

Estos resultados parecen indicar que la autoestima funcionaría como un recurso promotor de la salud y protector de la disfuncionalidad, emergiendo como un posible factor de resiliencia. Al respecto, Kobasa (1985) la menciona entre las características inherentes a las personalidades resistentes, y Grotberg (2006) la posiciona como un elemento importante dentro del factor resiliente "fuerza interior" o "yo soy". Melillo, Estamatti y Cuestas (2008) otorgan tal relevancia a la autoestima consistente que la consideran base de los demás pilares que sustentan la resiliencia en niños y adolescentes.

No obstante, debe tenerse en cuenta que la autoestima no es un artilugio mágico que permite por sí solo el mejoramiento de las capacidades de los individuos y el aumento de la probabilidad de una adaptación exitosa al medio. Es necesario reconocer que a menudo, si no siempre, su intervención positiva se ve potenciada por otros factores protectores con los que interactúa, de tal manera que la dinámica entre factores es, en ocasiones, más importante que la actuación de factores aislados (Grotberg, 1995).

\section{Las bases de la autoestima}

Las creencias que una persona llega a tener del contexto que la rodea involucran aspectos tanto cognitivos como afectivos, lo que lleva a pensar que la génesis y desarrollo del autoconcepto y la autoestima son procesos complementarios y continuos a lo largo del ciclo evolutivo de una persona. Ambas construcciones paralelas implicarán factores perceptivos y atribucio- 
nales en los que se dificulta diferenciar lo intrínseco del sujeto y la influencia pura de su ambiente más próximo (González Arratia y Gil Lacruz, 2006).

Si bien existen muchos enfoques teóricos que explican la estructuración y desarrollo del autoconcepto y de la autoestima, se conocen dos teorías psicológicas que destacan la importancia de la calidad de la parentalidad en la formación del concepto de sí mismo y su valoración: (a) el interaccionismo simbólico o teoría del espejo (Berger y Luckman, 1986) y (b) la teoría del aprendizaje social (Bandura, 1982).

Según la teoría del interaccionismo simbólico, las autovaloraciones se construyen a partir de la retroalimentación ofrecida por figuras significativas, por lo que son resultado de las percepciones del entorno próximo. La persona se ve reflejada en la imagen que le ofrecen otros, como si éstos fueran un espejo. Desde esta perspectiva, el niño llega a ser como los otros significativos piensan que es. En los primeros años de vida, la información de sí mismo se recibe casi exclusivamente de los padres. No obstante, con los años se incorporan otras figuras significativas como lo son profesores, compañeros de escuela y amigos.

Por otra parte, la teoría del aprendizaje social de Bandura (1982) sugiere que el niño forma su autoconcepto a partir de un proceso de imitación en el que incorpora actitudes y comportamientos de las personas significativas, especialmente de los padres.

En climas familiares sanos, los niños son sujetos activos, por lo que sus propias actitudes y experiencias también tienen un peso significativo en la conformación de su autoconcepto y autovaloración. Harter (1983) señala que la autoestima y el autoconcepto de los niños proviene de dos fuentes primordiales: (a) el apoyo que perciben de las demás personas y (b) cuán competentes se sienten los niños en diferentes ámbitos. No obstante, la autora sugiere que la contribución principal es el respeto de las personas más significativas en sus vidas, es decir, sus padres y familiares, seguidos de docentes y amigos.

Numerosas investigaciones empíricas llevadas a cabo con niños y adolescentes confirman que la familia es una pieza clave para la génesis de la autoestima. De todas las condiciones familiares que pueden afectar la valoración personal de los hijos, los aspectos afectivos y relacionales parecen ocupar un lugar más privilegiado que las características formales de las familias, tales como cantidad de hermanos, la constitución familiar, el orden de nacimiento, etc. (Navarro, et. al 2006; Portillo Estrada y Torres Velazquez, 2007).

Cuanto más positivamente se percibe la relación familiar más elevada tiende a ser la autoestima de los hijos (Peixoto, 2004). Espinoza y Balcazar 
(2002), en una investigación realizada con niños maltratados y niños de familias caracterizadas por buenos tratos, encontraron que los niños de familias con buen ambiente mostraban una autoestima más elevada que los menores maltratados. En contraste, las vivencias negativas en el hogar contribuyen a una autodefinición y autovaloración inadecuada del niño, tal como se señala en otros estudios (Natera, Mora, Nava, Tiburcio, 1994), ya que es frecuente que quienes han vivido en un ambiente hostil desarrollen una autoestima deficiente (González Arratia y Gil Lacruz, 2006; Cava, et. al, 2006, Ramírez Castillo, 2007).

Dentro de las características afectivas y relacionales de los padres que más contribuyen a desarrollar y fortalecer la autoestima positiva de los hijos se encuentran la aceptación y el apoyo incondicional. Los estudios de Coopersmith $(1967,1968)$, por ejemplo, mostraron que los niños/adolescentes con alto aprecio a sí mimos eran hijos de madres con elevada autoestima, que aceptaban y brindaban apoyo a sus hijos. Más recientemente, MartínezFerrer, Murghi Pérez, Musitu Ochoa y Monreal Gimeno (2008), encontraron que el apoyo parental (comprendido por conductas de soporte emocional e informacional, respeto por la autonomía y aceptación del hijo como persona) correlacionaba positivamente con la autoestima escolar y familiar, tanto de niños como de adolescentes. En contraposición, un estudio realizado en universitarios mostró una alta relación entre la percepción de desaprobación parental y la autoestima negativa en los hijos Los hijos que se sienten rechazados por sus padres (especialmente por la madre) ven afectada su autovaloración (Sansinenea y Sansinenea, 2004).

Relacionado con la aceptación, la comunicación familiar adecuada también favorece el desarrollo de la autoestima en los hijos (Cava, et. al, 2006). En particular, el contenido emocional de las conversaciones que se desarrollan entre padres e hijos, con especial énfasis en las evaluaciones y emociones positivas, es un elemento que parece estar asociado significativamente a la autovaloración de los niños (Reese, Bird y Tripp, 2007).

La aceptación parental es ampliamente reconocida como una de las dimensiones básicas que constituyen los diferentes estilos de crianza. Diversos investigadores han señalado que existe una estrecha relación entre los estilos educativos y diferentes áreas del desarrollo humano asociados con el nivel de autoestima (Coopersmith, 1967; Cassidy, 1990; Alonso García, 2002; Barudy y Dantagnan, 2005). Las características del estilo parental incluyen dos aspectos básicos y relacionados: (a) la demostración y comunicación del afecto en la relación (aceptación- rechazo, calor-frialdad, afecto-hostilidad, 
proximidad-distanciamiento) y (b) las acciones puestas en juego para controlar y disciplinar el comportamiento infantil (control y disciplina). A partir de las mencionadas dimensiones básicas, la propuesta inicial de Baumrind (1971) y los aportes posteriores de Macoby y Martin (1983) han dado lugar a una de las tipologías parentales más conocidas: democrático, autoritario, permisivo y negligente. Estudios posteriores, como el aportado por Palacios y Rodrigo (1999), dan cuenta de que los hijos de padres democráticos (equilibrados en control y aceptación-afectividad) se destacan por su competencia social, por su elevada autoestima, por su capacidad de autocontrol, por su mayor autonomía y baja impulsividad. Los hijos de padres permisivos (bajos en control y altos en aceptación-afectividad) son alegres, vitales, creativos y con autoestima elevada, sin embargo, muestran dificultades para el control de sus impulsos, la constancia en la finalización de las tareas y son más sensibles a las presiones por parte del grupo de pares.

Resultados similares han sido informados por Alonso García y Román Sánchez (2005), quienes encontraron una relación significativa entre los estilos parentales y la autoestima de niños pequeños ( 3,4 y 5 años de edad). Los autores arribaron a la conclusión de que un estilo de disciplina equilibrado en control y aceptación favorece la autoestima, mientras que un estilo autoritario (alto en control y bajo en aceptación) la debilita. En este estudio, los resultados con respecto al estilo permisivo no arrojaron datos unánimes con relación a ambos progenitores, ya que mientras la permisividad del padre parecía aumentar la autoestima de los niños, la de la madre tendía a debilitarla.

Investigaciones más recientes han llegado conclusiones semejantes al concluir que los estilos de crianza autoritativos o equilibrados favorecen valores de autoestima más elevados que los estilos autoritarios, negligentes, indulgentes y permisivos; y que en general, de éstos cuatro últimos, el estilo permisivo, es el que sigue en orden de importancia predictiva, también en sentido positivo (Martínez y García, 2007; Milevsky, Schlechter, Netter y Keehn, 2007).

Resumiendo lo expuesto hasta aquí, se puede afirmar que "lo que los padres sienten, piensan y hacen por sus hijos y la forma en que lo comunican impacta en la manera en que los hijos se conciben a sí mismos" (Barudy y Dantangnan, 2005, p.87). Los progenitores reflejan cómo se sienten en presencia de un hijo y esto es internalizado por el niño, actualizándose y reforzándose en cada nueva experiencia. Dichos mensajes están íntimamente relacionados con lo que el niño sentirá por sí mismo. La parentalidad adecuada se caracteriza, entonces, por facilitar el desarrollo de una identidad sana 
y una autoestima elevada. Por el contrario, los padres que no poseen dichas capacidades, envían mensajes negativos a sus hijos, lo que explica un autoconcepto negativo y una baja autoestima.

Las investigaciones analizadas han destacado la influencia de las figuras significativas en la conformación de la autoestima infantil, especialmente los padres o cuidadores de los niños, de lo que se desprende la importancia de estudiar los primeros entornos sociales como predictores de la configuración del autoconcepto y del nivel de autovaloración (Cassidy, 1990; Cichetti, Rosch, Toth, \& Spagnola, 1997; Alonso García, 2002; Alonso García \& Román Sánchez, 2005).

El apoyo psicosocial en beneficio de los recursos familiares: Programas para padres

Tal como ha sido expuesto en los estudios citados anteriormente, la familia se constituye como el primer contexto de formación de la autoestima y de particular influencia en las primeras etapas del desarrollo humano, por lo que requiere especial deferencia.

Para muchos autores existe un cierto consenso respecto de las funciones de la familia en lo que a la crianza de los hijos concierne: (a) satisfacer sus necesidades físicas y materiales, es decir, asegurar su supervivencia y un crecimiento sano; (b) suplir sus necesidades emocionales y sociales creando el clima de afecto y apoyo emocional necesario para un desarrollo psicológico saludable; (c) contribuir a la satisfacción de necesidades cognitivas, aportándoles la estimulación que haga de ellos seres con capacidad para adaptarse de modo competente a su entorno físico y social; y (d) tomar decisiones respecto a la apertura hacia otros contextos educativos que van a compartir con la familia la tarea de educación y socialización del niño o la niña, destacándose entre éstos, el ámbito escolar (Barudy \& Dantagnan, 2005; Bringiotti, 2005; Palacios \& Rodrigo, 1998).

En este sentido, algunas investigaciones afirman que una de las funciones psicológicas más importantes que atañe a la familia es la formación del autoconcepto y de la autoestima como parte de la identidad de los hijos (Musitu y Allatt, 1994; Alonso García \& Roman Sánchez, 2005).

Muchos estudios psicosociales han apuntado el debilitamiento de estas funciones familiares en la sociedad actual (Bringiotti, 2005; Bolivar, 2006; Walsh, 2004). Aunque cada familia es única y diferente en sus formas, capacidades y posibilidades para llevar a cabo estos objetivos, es importante destacar que "hay un límite que se coloca en el momento y situación en 
que se vea alterado el bienestar y crecimiento de los hijos" (Bringiotti, 2005, p.82), es decir, cuando los derechos de los niños se encuentran vulnerados y sus necesidades insatisfechas, poniendo en riesgo su adecuado desarrollo integral (físico, emocional, cognitivo y social). Lamentablemente, el quiebre de los límites que aseguran un propicio desarrollo infantil es una realidad que ha tenido un vertiginoso aumento en los diversos escenarios familiares y sociales, generando situaciones que ponen en riesgo a muchos niños y niñas (negligencia, abandono físico y afectivo parental, maltrato físico y psicológico, abuso sexual infantil intrafamiliar, entre otras).

A la compleja realidad social actual, debemos sumarle las reflexiones de algunos autores que señalan que: "la familia es la única institución educativa en la que sus miembros adultos no reciben preparación específica, que mejore y optimice su competencia educativa" (Maiquéz, Rodrigo, Capote y Vermaes, 2000, p.9). En vista de esta necesidad de fortalecimiento familiar, surgen los programas de "educación para padres" o "escuelas para padres" desde un innovador enfoque terapéutico y psicoeducativo que consiste en capacitar a madres, padres y/o otros cuidadores en principios, técnicas y estrategias que les permitan mejorar sus prácticas parentales (Rey, 2006). Dichos programas se fundan en la premisa de algunos autores (Máiquez, Rodrigo, Capote y Vermaes, 2000; Rey, 2006; Sanders y Morawska, 2010) que aseguran que los padres necesitan formarse para hacer frente a los desafíos sociales que requiere la función de crianza en la actualidad. Muchas de estas intervenciones están basadas en una perspectiva ecosistémica, con el fin de generar condiciones de apoyo social adecuadas para que los padres logren afianzar sus propios recursos y ejercer una apropiada parentalidad social (Barudy y Dantagnan, 2005; Gracia, 1997; Vargas Rubilar y Oros, inédito). En las realidades sociales presentes, el entrenamiento parental es clave, ya que constituye una acción formativa de sensibilización, de orientación o de clarificación de los valores, prioridades, actitudes y prácticas de los padres en la educación de sus hijos (Rey, 2006; Sanders y Morawska, 2010).

La mayoría de estas valiosas iniciativas de intervención familiar, comparten como objetivo principal prevenir y abordar una variedad de problemas socio-familiares que se encuentran asociados a la falta de apoyo y educación de los padres como principales responsables de las familias y del desarrollo emocional y social de sus hijos (Pérez Campos, 2005). Por este motivo, los programas para padres se presentan como una alternativa de abordaje idónea para la promoción de la autoestima infantil, y de tantos otros recursos psicológicos necesarios para un adecuado ajuste psicosocial de niños y jóvenes. 


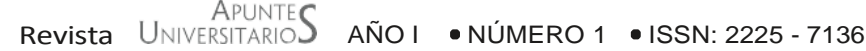

\section{Consideraciones finales}

En el presente trabajo se ha destacado el irremplazable rol que cumple el contexto familiar en el desarrollo de la autoestima infantil, por lo que dicho ámbito requiere especial cuidado desde los distintos ámbitos de intervención preventiva y asistencial.

Si bien la mayor parte de la literatura sobre resiliencia individual y familiar señala la importancia vital de la función de los padres: modelar y guiar a los niños en una dirección positiva, facilitando el desarrollo de una identidad sana y una autoestima elevada; desdichadamente, esto no siempre es llevado a la práctica, por lo que es impostergable generar acciones específicas para fortalecer las competencias familiares. Es allí donde deberían centrarse muchos de los esfuerzos de la intervención psicológica y social, tanto en el ámbito clínico como en el comunitario. Por otra parte, los enfoques actuales han prestado atención además al importante papel que pueden desempeñar otros recursos sociales: la familia extensa, la escuela, los centros de salud, los centros de actividades extraescolares, entre otros, en favor de los grupos familiares (Gómez, Muñoz y Haz, 2007, Walsh, 2004).

La autoestima positiva es un constructo importante a promover a nivel familiar, no sólo porque incluye aspectos personales o individuales, sino por la influencia que ejerce a nivel social en los individuos: "Las personas se evalúan a sí mismas a partir de los éxitos y fracasos cotidianos, en comparaciones sociales con los demás y en comparaciones con sus propios parámetros internos" (Sanchez Santa- Bárbara, 1999, p. 252). Tal vez esta sea una de las razones por la que la autovaloración ha sido considerada clave en muchos modelos de terapia familiar que han mostrado ser efectivos y reconocidos en el área de la Psicología clínica y comunitaria (Grotberg, 2006; Neil y Neil, 2001; Satir, 1991; Walsh, 2004).

La adopción de un enfoque positivo de base en pro de la autoestima por parte de los interventores de programas para padres, puede hacer una notable diferencia en las familias atendidas. Aunque las familias son sistemas dinámicos, deben generar cambios que aseguren el crecimiento y bienestar de sus miembros. La perseverancia, la esperanza, el aliento y el optimismo, son elementos esenciales a la hora de fortalecer recursos y de promover cambios estructurales, sobretodo si esto significa desaprender algunas prácticas parentales heredadas de las familias de origen y arraigadas por generaciones (Walsh, 2004). 
Como reflexión final podemos apuntar que, como sociedad, transitamos en épocas de grandes cambios sociales y por ende, los profesionales hemos sido convocados a nutrir y reforzar a las familias con nuevas estrategias desde las distintas áreas del quehacer educativo y de la salud (mental y física). Es tiempo de reconectarnos con este desafío, como una realidad que nos pertenece, una realidad de la todos somos responsables.

Jael Alejandra Vargas Rubilar Universidad Adventista del Plata e-mail: cipca9@uapar.edu

Laura Beatriz Oros

Universidad Adventista del Plata e-mail: fachuin2@uapar.edu

Recibido: 10 de Noviembre de 2011 Aceptado: 25 de Noviembre de 2011

\section{Referencias}

Abela, J. R. Z. \& Skitch, S. A. (2007). Dysfunctional attitudes, self-esteem, and hassles: Cognitive vulnerability to depression in children of affectively ill parents. Behaviour Research and Therapy, 45 (6), 1127-1140.

Alonso García, J. (2002). Práctica educativas familiares y autoconcepto. Estudio con niños y niña de 3, 4, y 5 años- . Universidad de Valladolid. Departamento de Psicología. Tesis doctoral.

Alonso García, J. \& Román Sánchez, J. M. (2005). Prácticas educativas familiares y autoestima. Psicothema, 17 (1), 76-82.

Bandura, A. (1982). Aprendizaje Social. Madrid: Espasa- Calpe.

Barudy, J. \& Dantagnan, M. (2005). Los buenos tratos a la infancia: Parentalidad, apego y resiliencia. Barcelona: Gedisa.

Baumrind, D. (1971). Current patterns of parental authority. Devolopmental Pyschology, 4, 1-103.

Berger, M. \& Luckman, T. (1986). La construcción social de la realidad. Buenos Aires: Amorrortu. 


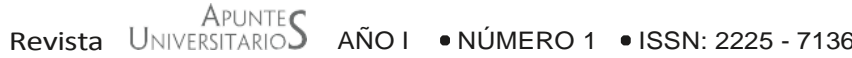

Bolivar, A. (2006). Familia y escuela: dos mundos llamados a trabajar en común. Revista de Educación, 339, 119-146.

Bragado, C., Hernández LLoreda, M. J., Sánchez-Bernardos, M. L. \& Urbano, S. (2008). Autoconcepto físico, ansiedad, depresión y autoestima en niños con cáncer y niños sanos sin historia de cáncer. Psicothema, 20 (3), 413-419.

Bringiotti, M. (2005). Las familias en situación de riesgo en los casos de violencia familiar y maltrato infantil. Texto y contexto- Enfermagem, 14, 78-85.

Cassidy, J. (1990). Theorical and methodological considerations in the study of attachment and the sefl in young children. En M. T. Greenbers, D. Cichetti y E. M. Cummings (Eds). Attachment in the preschool years, theory, research and intervention (pp. 87-118). Chicago: University of Chicago.

Cardenal, V. \& Fierro, A. (2004). Componentes y correlatos del autoconcepto en la escala de PiersHarris. Estudios de psicología, 24 (1), 101-111.

Cava, M., Musitu, G. \& Murgui, S. (2006). Familia y violencia escolar: el rol mediador de la autoestima y la actitud ante la autoridad institucional. Psicothema, 18, 3, 367-373.

Ciarrochi, J., Heaven, P. C. L., \& Davies, T. (2007). The impact of hope, self-esteem, and attributional style on adolescents' school grades and emotional well-being: A longitudinal study. Journal of Research in Personality, 41 (6), 1161-1178.

Cichetti, D., Rogosch, F.A., Toth \& Spagnola, M. (1997). Affect cognition and emergence of self. knowledge in the toddler offspring of depressed mothers. Journal of Experimental Child Pyschology, 67, 338-362.

Coopersmith, S. (1967). The antecedents of self-esteem. San Francisco: Freeman and Company.

Coopersmith, S. (1968). Studies in self -esteem. Scientific American, 218, 96-106.

Diamantopoulou, S., Rydell, A. M. \& Henricsson, L. (2008). Can Both Low and High Self-esteem Be Related to Aggression in Children? Social Development, 17 (3), 682-698.

Espinoza, A. \& Bacazar, P. (2002). Autoconcepto y autoestima en niños maltratados y en niños con familias intactas. Psicología.com, 2002; 6, 1.

Falcone Castillo J., Castro Serran, B., Serrano Pérez, P., Ortiz Ramírez,V., Toral Chaigneau, M, Vuskovic Gatica, C. \& Guzmán Valencia, C.(2009). Promoción de la calidad de vida: Evaluación de impacto del programa "Fortalecimiento para una vida saludable. International Journal of Psychological Research, 2, 1, 24-34.

Fiorentino, M.T. (2008) La construcción de la resiliencia en la calidad de vida y de la salud. Suma Psicológica, 15 (1), 95-114. 
Fredrickson, B. L. (2004). Gratitude, like other positive emotions, broadens and builds. In Emmons, R. A. \& M. E. McCullough (Eds.) The Psychology of Gratitude. (pp. 145-166). New York: Oxford University Press.

Frías, D., Mestre, V., \& Del Barrio, V. (1990): Revisión histórica de los conceptos de de-presión y self. Revista de Historia de la Psicología, 10, 291-297

Garaigordobil, M. \& Durá, A. (2006). Relaciones del autoconcepto y la autoestima con la sociabilidad, estabilidad emocional y responsabilidad en adolescentes de 14 a 17 años. Análisis y modificación de conducta, 32 (141), 37-64.

Garaigordobil, M.; Pérez, J. I. \& Mozaz, M. (2008). Self-concept, self-esteem and psychopathological symptoms Psicothema, 20 (1), 114-123.

Gázquez, J. J.; Pérez, M. C.; Ruiz, M. I.; Miras F. \& Vicente, F. (2006). Estrategias de aprendizaje en estudiantes de enseñanza secundaria obligatoria y su relación con la autoestima. International Journal of Psychology and Psychological Therapy, 6 (1),51-62.

Gómez, E., Muñoz, M. \& Haz, A. (2007). Familias multiproblemáticas y en riesgo social: Características e intervención. Psykhe, 16, (2), 43-54.

González Arratia, N. \& Gil La Cruz, M. (2006). Autoestima y socialización maternal: un análisis transcultural. Persona, 9, 189-201.

González-Pienda, J. A.; Núñez Pérez, J. C.; Glez.-Pumariega, S. \& García García, M. S. (1997). Autoconcepto, autoestima y aprendizaje escolar. Psicothema, 9 (2), 271-289.

Gracia, E. (1995). El rol del apoyo social en la prevención del maltrato infantil: programas de apoyo a la familia. Intervención Psicosocial, 4 (10), 17-30

Greco, C.; Morelato, G. \& Ison, M. (2006). Emociones positivas: una herramienta para promocionar los procesos de resiliencia infantil. Psicodebate 7. Psicología, Cultura y Sociedad. Psicología positiva. Universidad de Palermo (7): 81 - 94

Grotberg, Edith (1995). The Internacional Resilience Proyect: Promoting Resilience in Children. Informe Técnico de Investigación. Alabama University, Civitan International Research Center. Recuperado de la www. http://eric.ed.gov/ERICWebPortal/search/ detailmini.jsp?_nfpb=true\&_\&ERICExtSearch_SearchValue_0=ED383424\&ERICExtSear ch_SearchType_0=no\&accno=ED383424

Grotberg, E. (2006). La resiliencia en el mundo de hoy. Barcelona: Gedisa.

Hart, D., \& Matsuba, M.K. (2007). The development of pride in moral life. In J.L. Tracy, R.W. Robins, \& J.P. Tangney (Eds.), The selfconscious emotions: Theory and research (pp. 114133). New York: Guilford. 


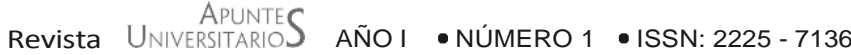

Harter, S. (1983). Developmental perspectives on the self-system. In P. H, Musen (Ed.). Handbook of Chile Psychology. New York. Wiley.

Heatherton, T. F., \& Wyland, C. L. (2003). Assessing self esteem. In S. J. Lopez \& C. R. Snyder (Eds.), Positive psychological assessment: Handbook of models and measures (pp. 219-233). Washington DC: American Psychological Association.

Hewitt, J. P. (2005). The social Construction of Self-Esteem. In C. R. Snyder \& S. J. López (Eds.), Handbook of Positive Psychology (135-147). New York: Oxford University Press.

Kobasa, S. (1985). Stressful life events, personality, and health: An inquiry into hardiness, en A. Monat y R. Lazarus, eds. Stress and coping, Segunda edición, Nueva York: Columbia University Press.

Maccoby, E.E \& Martín, J.A. (1983). Socialization in the context of the family: parent. Chiled interaction. En P. H. Mussen y E. M. Hetherington (Eds): Handbook of child psychology: socialization, personality and social development (pp.1-102) (vol. IV). New York: J.Wiley.

Maiquéz, M., Rodrigo, M., Capote, C., \& Vermaes, I. (2000). Aprender en la vida cotidiana, Un programa experiencial para padres. Madrid: Visor

Martínez-Antón, M., Buelga, S., \& Cava, M. J. (2007). La satisfacción con la vida en la adolescencia y su relación con la autoestima y el ajuste escolar. Anuario de Psicología, 38 (2), 293-303.

Martínez-Ferrer, B., Murgui-Pérez, S., Musitu-Ochoa, G. \& Monreal-Gimeno, M. C. (2008). El rol del apoyo parental, las actitudes hacia la escuela y la autoestima en la violencia escolar en adolescentes. International Journal of Clinical and Health Psychology, 8 (3), 679-692.

Martínez, I. \& García, J. F. (2007). Impact of Parenting Styles on Adolescents' Self-Esteem and Internalization of Values in Spain. The Spanish Journal of Psychology, 10 (2), 338-348.

Melillo, A., Estamatti, M. \& Cuestas, A. (2008). Algunos fundamentos psicológicos del concepto de resiliencia. En A. Melillo y E. N. Suárez Ojeda (Comp.), Resiliencia, descubriendo las propias fortaleza (pp. 83-102). Buenos Aires: Paidós.

Milevsky, A., Schlechter, M., Netter, S. \& Keehn, D. (2007). Maternal and Paternal Parenting Styles in Adolescents: Associations with Self-Esteem, Depression and Life-Satisfaction. Journal of Child and Family Studies, 16, 39-47.

Mruk, C. J. (2006). Self-esteem Research, Theory and Practice. New York: Springer.

Musitu,G., Allatt, P. (1994). Psicología de la Familia. Valencia: Albatros 
Musitu, G., Jiménez, T. I. \& Murgui, S. (2007). Funcionamiento familiar, autoestima y consumo de sustancias en adolescentes: un modelo de mediación Salud pública de México, 49 (1), 3-10.

Nataera, G., Mora, S., Nava, A. \& Tiburcio, M. (1994). Violencia familiar y su relación con la farmacodependencia. México: Elpac/Amepso.

Neil, S.E.S. \& Neil, S. E (2001). A jorney througth three continents and four generations: A family reconstruction. Melbourne: Satir centre of Australia.

Navarro, E., Tomás, J. M. \& Oliver, A. (2006). Factores personales, familiares y académicos en niños y adolescentes con baja autoestima. Boletín de psicología, 88, 7-25.

Oros, L.B. \& Richaud de Minzi, M.C. (en prensa). La promoción de emociones positivas en la escuela. Propuestas para integrar el desarrollo afectivo al currículo escolar. Entre Ríos: Universidad Adventista del Plata.

Orth, U., Robins, R. W. \& Roberts, B. W. (2008). Low Self-Esteem Prospectively Predicts Depression in Adolescence and Young Adulthood. Journal of Personality and Social Psychology, 95 (3), 695-708.

Palacios, J. y Rodrigo, M. (1999).Familia y desarrollo humano. Madrid: Alianza Editorial.

Peixoto, F. (2004). Qualidade das relações familiares, auto-estima, autoconceito e rendimento académico. Análise Psicológica, 1 (22), 235-244.

Pérez Campos, G. (2005). La complejidad de los marcos de interacción en educación de padres. Avances en Psicología Latinoamericana 23, 177-192.

Portillo Estrada, C. J. \& Torres Velazquez, L. E. (2007). Efectos en la crianza de familias uniparentales: La autoestima. Revista Electrónica de Psicología Itzacala, 10 (1), 16-38.

Reese, E., Bird, A. \& Tripp, G. (2007). Children's Self-esteem and Moral Self: Links to Parent-Child Conversations Regarding Emotion. Social Development, 16 (3), 460-478.

Rey, C. (2006). Entrenamiento de padres: una revisión de sus principales componentes y aplicaciones. Revista Infancia, Adolescencia y Familia, 1, 61-84.

Ramírez Castillo, M. Los padres y los hijos: variables de riesgo. Educación y educadores, 10,1, 27-37

Ramírez Peradotto, P.; Duarte Vargas, J. \& Muñoz Valdivia, R. (2005). Autoestima y refuerzo en estudiantes de 5o básico de una escuela de alto riesgo. Anales de Psicología, 21 (1), 102-115. 


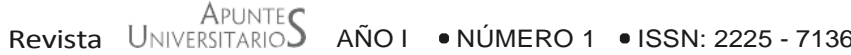

Rutter, M.(1985). Resilience in the face of adversity: Protective factors and resistance to psyquiatric disorder. British Journal of Psychiatry, 147, 598-611.

Sánchez Santa-Bárbara. E. (1999). Relación entre la autoestima personal, la autoestima colectiva y la participación en la comunidad. Anales de Psicología, 15 (2), 251-260.

Sanders M., Morawska A. (2010) ¿Es posible que el conocimiento de los padres, las competencias y expectativas disfuncionales, y la regulación emocional mejoren los resultados de los niños? In: Tremblay RE, Barr RG, Peters RDeV, Boivin M, eds. Enciclopedia sobre el Desarrollo de la Primera Infancia [en línea]. Montreal, Quebec: Centre of Excellence for Early Childhood Development; Recuperado el 10 de Enero de 2011 de: http://www.enciclopedia-infantes.com/documents/Sanders-MorawskaESPxp.pdf.

Sansinenea, P. y Sansinenea, E. (2004). Autoesti y desaprobación parental. Psiquis, 25(4):167-175.

Satir, V. (1991) The Satir Model. Palo Alto, CA: Science and behavior books

Seligman, M. (1998). Fuerza humana constructiva. Misión olvidada de la Psicología. APA: Monitor 29, 1.

Vargas Rubilar, J. A. \& Oros, L. B. (inédito). Una propuesta de intervención psicoeducativa para promover la autoestima infantil. Centro de Investigación en Psicología y Ciencias Afines, Universidad Adventista del Plata.

Verduzco Álvarez-Icaza, M. A. (2006). La autoestima, estrés y afrontamiento desde la perspectiva de niños y niñas escolares. Hologramática, 2 (5), 59-70.

Walsh, F. (2004). Resiliencia familiar: Estrategias para su fortalecimiento. Buenos Aires: Amorrortu

Williams, L. A. \& DeSteno, D. (2008). Pride: Adaptive Social Emotion or Seventh Sin? Psychological Science, 20 (3), 284-288. 